\title{
Review
}

\section{Purinergic signaling: a novel mechanism in immune surveillance}

\author{
Lin $\mathrm{MEI}^{1}$, Wei $\mathrm{DU}^{1}$, Wei $\mathrm{GAO}^{1, *}$, Qi-bing $\mathrm{MEI}^{2, *}$ \\ ${ }^{1}$ Department of Cardiology, Peking University Third Hospital, Peking University Health Science Center, Beijing 100191, China; \\ ${ }^{2}$ Department of Pharmacology, School of Pharmacy, Fourth Military Medical University, Xi'an 710032, China
}

\begin{abstract}
Purinergic receptors and the associated signaling cascades are known to play critical roles in cardiovascular, nervous, respiratory, gastrointestinal and urinogenital systems. Recent studies have also shed light on the importance of nucleotides and purinergic receptors in the regulation of the immune response. With a better understanding of the distribution and the receptor subtypes, the purinoceptors have the potential to become important therapeutic targets in inflammation, chemotaxis and immune-related diseases.
\end{abstract}

Keywords: nucleotides; purinergic receptor; immune surveillance; inflammation; chemotaxis

Acta Pharmacologica Sinica (2010) 31: 1149-1153; doi: 10.1038/aps.2010.128; published online 16 Aug 2010

\section{Introduction}

Extracellular nucleotides and their metabolites are important signaling molecules that enact various biological effects through specific receptors. The receptors for these molecules can be divided into two major families: the P1 receptor for adenosine and the P2 receptor for ATP, ADP, UTP, and UDP ${ }^{[1]}$. Based on differences in structure and transduction mechanism, P2 receptors can be sub-classified as ligand-gated ion channel receptors and G protein-coupled receptors (GPCRs), referred to as $\mathrm{P} 2 \mathrm{X}$ and $\mathrm{P} 2 \mathrm{Y}$ receptors, respectively. To date, seven P2X and nine P2Y subtype receptors have been cloned, and these receptors are widely distributed in various mammalian organs. All immune and inflammatory cells express P2Y and P2X receptors, and their expression is modulated by inflammatory cytokines during development. Among the P2 receptors, P2Y2, P2Y6, P2Y11, P2Y12, P2X4, and P2X7 are closely associated with immune responses.

In particular, the physiological role of $\mathrm{P} 2 \mathrm{X} 7$ on the release of IL-1 $\beta$ has been clearly demonstrated ${ }^{[2]}$. During infection, tissue injury, inflammation, tumor immunity and apoptosis, nucleotides are released or secreted from cells, contributing not only to host defense but also to phagocytic clearance. Thus, both modulation of the release of these nucleotides and influence on the receptors represent important therapeutic strategies for the treatment of immune-related diseases.

\footnotetext{
* To whom correspondence should be addressed.

E-mail weigao@bjmu.edu.cn (Wei GAO); qbmei@fmmu.edu.cn (Qi-bing MEl)

Received 2010-06-04 Accepted 2010-07-12
}

\section{P2 receptors and chemotaxis}

It has long been known that the activation of phagocytic cells is accompanied by ATP release. However, the specific receptor and signaling are still unknown. Neutrophils, the leukocytes that play a central role in host defense by destroying microorganisms, also secrete ATP when they become activated or start clearing dying cells. Further, it was found that ATP was released predominantly from the protruding regions at the leading edge of the migrating neutrophils. Hydrolysis of ATP by apyrase or CD39, which hydrolyze ATP to AMP, strongly affects chemotaxis, suggesting that the hydrolysis of ATP is crucial in the initiation of migration ${ }^{[3]}$. Early reports speculated that activation of $\mathrm{P} 2 \mathrm{X} 7$ receptors was one of the most efficient stimuli for IL- $1 \beta$ release $^{[4]}$. Chen reported that P2Y2 and A3 receptors were concentrated at the leading edge of the polarized cells and concluded that P2Y2 receptors controlled cell orientation. Additionally, this group suggested that following the hydrolysis of ATP and the formation of adenosine, the A3 adenosine receptors further facilitated the speed of chemotaxis. This hypothesis was confirmed by studies in gene knockout mice ${ }^{[5]}$. Recently, Elliott et al. targeted a "find me" signal on extracellular nucleotides, and these signals potently induced monocyte activation both in mammalian cell culture and in mice. They used an air-pouch model, which allowed direct tracking of cell movement in vivo, and showed that apoptotic cells selectively attracted monocytes and macrophages. Further experiments identified P2Y2 as the specific receptor involved in the process of clearance. Enzymatic breakdown of ATP and UTP, pre-incubation with a P2Y2 inhibitor and use of gene knockout mice all impaired 
thymic apoptosis-induced chemotaxis ${ }^{[6]}$. For this reason, ATP seems not only to activate macrophages but also to regulate some scavenging functions. For instance, the chemotaxis and degranulation of mast cells are also mediated by P2X7Rinduced calcium release ${ }^{[7]}$.

Microglia are the principal immune cells in the brain. Previous studies found that microglial processes rapidly converged on the site of injury, isolating normal from injured tissue. The motility of these cells can be inhibited by ATP-hydrolyzing enzymes or P2Y receptor inhibitors ${ }^{[8]}$. Although the specific subtype of P2Y receptors are yet to be identified, the report from Haynes et al. showed that microglia from $\mathrm{P}_{2} \mathrm{Y}_{12} 2^{-/-}$mice were unable to polarize, migrate or extend processes toward nucleotides. Moreover, P2Y12 receptor expression was high basally but dramatically reduced after activation, indicating that P2Y12 receptors may initiate the early stage of the microglial respons $\mathrm{e}^{[9]}$. Furthermore, the platelet P2Y12 receptor also mediates morphological changes, suggesting that clopidogrel (a P2Y12 inhibitor) may have an extra-neuroprotective role. In addition to ATP, UTP and other nucleotides may also play important regulatory roles, as indicated by a more recent report from Koizumi ${ }^{[10]}$. Following injury, the P2Y6 receptor was up-regulated with concurrent down-regulation of P2Y12, and this event triggered phagocytosis. Koizumi et al also found that after chemical injury of the hippocampal region in vivo, the levels of UTP increased. It was speculated that UTP and its degradation product, UDP, mediated the unique functions of microglia: ATP regulates motility through the P2Y12 receptor using a "find me" signal, whereas UTP triggers phagocytosis through its specific P2Y receptor by an "eat me" signal ${ }^{[11]}$. In other words, ATP releases a kind of diffuse signal, orientating the phagocyte, while UTP and UDP work more locally to accurately guide the phagocytic process. This hypothesis was fully supported by a recent report ${ }^{[12]}$. The activation of phagocytes must be stringently regulated because the body must be protected from an unwanted response by these cells. Purinergic signaling serves as one of the crucial parts in this complicated crosstalk (see Figure 1). Developing new agents that target the purinergic signaling system may prevent unwanted inflammatory responses.

\section{P2 receptors and inflammation}

Normally, inflammation is triggered by invading microorganisms via stimulation by various cytokines. Atarashi et al. reported that bacteria could activate a unique subset of colon lamina propria cells through an increase in luminal ATP concentration, leading to the differentiation of $\mathrm{T}_{\mathrm{H}} 17$ cells, which are crucial in the pathogenesis of colitis ${ }^{[13]}$. However, it is also known that cell stress or tissue damage can initiate inflammation even in the absence of pathogens. Compelling evidence demonstrated that immune cells responding to injury release molecules that are normally located inside the cell. Among these "damage-associated molecular patterns," ATP has all the right features: its concentration is high within the cytoplasm and low in the extracellular space, and it is quickly released after cell damage, rapidly inactivated by ecto-ATPases and further regulated by the adenosine receptor ${ }^{[14]}$.

Accumulation of ATP in the extracellular space generates a dual activity. Moderate ATP release causes mainly antiinflammatory effects. Here, ATP activates P2Y11 receptors, causing maturation of dendritic cells (DCs) and the induction of Th2 cells ${ }^{[15]}$, or it facilitates T-cell activation through P2X7mediated calcium influx ${ }^{[16]}$. However, the release of ATP at high concentrations brings other $\mathrm{P} 2$ receptors into play, leading to a massive release of pro-inflammatory mediators and initiating the process of apoptosis. For example, high ATP accumulation in the airways in an asthmatic animal model could enhance Th2 sensitization, resulting in over-activated lung myeloid DCs ${ }^{[17]}$. Special attention should be paid to the P2X7 subtype, as it is closely related to IL-1 $\beta$ and IL-18. IL-1 $\beta$ is a potent pro-apoptotic agent that can be driven by P2X7 activation rather than by a passive process, as previously thought.

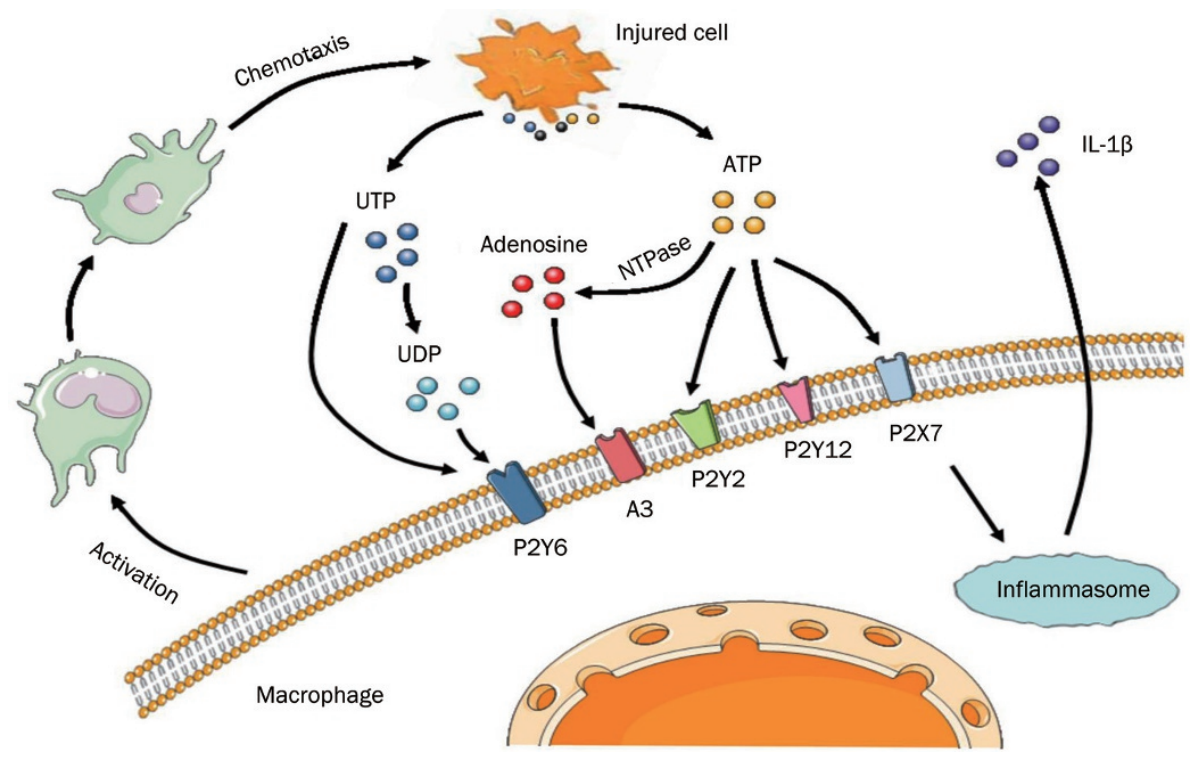

Figure 1. Purinergic signaling regulates phagocyte migration. 
It is possible that $\mathrm{P} 2 \mathrm{X} 7$ activation simultaneously opens the potassium channel and facilitates IL-1 $\beta$ secretion $^{[18]}$. For example, monocytes from patients with rheumatoid arthritis were more sensitive to release of IL- $1 \beta$ following ATP activation of the P2X7 receptor ${ }^{[19]}$. Mice deficient in $\mathrm{P} 2 \mathrm{X} 7$ exhibited markedly reduced inflammation, pain and IL-1 $\beta$-mediated IL-6 production ${ }^{[20]}$. On the other hand, it is also possible that there may be a subset of pro-inflammatory cells, such as P2X7expressing DCs and natural killer cells (NK), that are exquisitely sensitive to ATP-induced apoptosis. Hence, high levels of ATP could drive these cells out of the inflammatory milieu, causing immuno-suppressive activity ${ }^{[21]}$.

The inflammasome, a caspase-activating platform, consists of a central scaffold protein that coordinates a number of additional proteins ${ }^{[2]}$. These proteins allow interactions with inflammatory caspases. Following inflammatory stimuli, caspase- 1 converts pro-IL- $1 \beta$ to IL- $1 \beta$. Recent studies found that the P2X7 receptor activates the $\mathrm{K}^{+}$current and panx-1, a member of the pannexin family, through a protein-protein interaction, suggesting that P2X7 is a key activator of the inflammasome ${ }^{[23]}$. Finally, nucleotides, adenosine and their metabolizing enzymes, CD39 and CD73, form a sophisticated surveillance network in immunomodulation. Studies on CD39, a recently discovered marker of Foxp $3^{+}$Treg cells, may lead to a new understanding of the role of purinergic signaling in the immune system ${ }^{[24]}$.

\section{P2 receptors and cancer}

Adenine nucleotides were first described as having anticancer activity in 1983. Recent evidence suggests that extracellular ATP inhibits human tumor growth, including cells from prostate, breast, colon and liver. In addition, ATP induces resistance of normal tissues to chemo- and radiation therapy and causes weight loss, anorexia and cachexia in older patients by expanding blood plasma ATP levels ${ }^{[25]}$. However, the mechanism by which ATP participates in these antitumor activities has yet to be resolved.

Multiple forms of P2 receptors have been studied in various types of cancer ${ }^{[26]}$. For example, mRNA for P2Y1, P2Y2, P2Y4, and P2Y6 receptors have been detected in melanomas. It is also known that the P2Y1 receptor stimulates a decrease, whereas the P2Y2 receptor subtype mediates an increase in cell number. The changes in cell number are likely to be regulated through PLC-coupled increases in intracellular $\mathrm{Ca}^{2+}$ levels. However, in some other type of cancer, such as esophageal and colorectal cancers, activation of P2Y2 receptors causes a decrease in cellular proliferation ${ }^{[27]}$. These seemingly contradictory data suggest that different second messenger systems may possess opposing effects in addition to the effects of ATP. Functional P2X7 receptors have been studied extensively in human cervical cancer cell lines. Treatment with a P2X7 antagonist led to cell apoptosis, possibly through the activities of caspase- 3 and caspase- 9 or by the IL-1 $\beta$ pathway ${ }^{[28]}$.

Several signaling pathways have been suggested as being coupled to the P2 receptor. The most common signaling pathway described begins with an increase in PLC activity, leading to Ins $(1,4,5) \mathrm{P} 3$ mobilization and $\mathrm{Ca}^{2+}$ release from the endoplasmic reticulum ${ }^{[29]}$. In addition, the P2Y receptor coupled to adenylyl cyclase affects cAMP levels. It is not clear at present whether any specific P2Y receptors activate or inhibit the production of cAMP in cancer cells. Clearly, this presents a novel challenge to develop effective agents selectively targeting cancer cells either through specific signaling pathways or by working in conjunction with other chemotherapy drugs.

\section{P2 receptors in cells of the CNS}

The central nervous system (CNS), an avascular tissue previously thought to be "immunoprivileged," is clearly involved in immune reactions. Recent reports revealed that ATP is not only a neurotransmitter but is also involved in glial proliferation, motility, survival, differentiation and myelination and in facilitating interactions between neurons. Presently, it is well known that purinergic signaling profoundly affects neuroinflammation $^{[30]}$.

In some cases, the release of ATP during brain injury is neuroprotective but in other circumstances, ATP contributes to the initiation of harmful immune reactions. Under normal physiological conditions, peri-neuronal ATP concentrations are maintained at a low level. However, brain injury can induce increased concentrations of ATP, triggering uncontrolled immune reactions. Various P2 receptors are expressed by astrocytes in both central and peripheral nerves. On one hand, reactive astrocytes can impede neuronal regeneration and synthesize toxic molecules. On the other hand, astroglial-mediated neuroinflammation can also be beneficial ${ }^{[2]}$. Among the mediators driving reactive astrogliosis, ATP has a prominent role. P2 receptors induce gliosis via extracellularregulated kinase (ERK) and protein kinase B/ Akt pathways ${ }^{[31]}$. Astrocytes can sense the severity of damage in the CNS by the concentration of ATP and can modulate tumor necrosis factor- $\alpha$ (TNF- $\alpha$ )-mediated inflammatory responses via P2 receptors $^{[32]}$. The specific activation of P2X7 in astrocytes facilitates the release of glutamate, GABA and ATP, which might influence the excitability of neurons under pathological conditions $^{[33]}$.

Microglia, the immune cells of the CNS, are also activated by nucleotides to release inflammatory cytokines, such as IL-1 $\beta$ and TNFa, and are involved in chemotaxis, as mentioned earlier. In addition, P2X7 receptors are up-regulated around amyloid- $\beta$ plaques in a transgenic model of Alzheimer's disease via superoxide production ${ }^{[34]}$. ATP can also selectively suppress the synthesis of inflammatory proteins through calcium influx via $\mathrm{P} 2 \mathrm{X} 7$ receptors. If pain is regarded as a sort of inflammation, blockade of $\mathrm{P} 2 \mathrm{X} 4$ receptors can reverse tactile allodynia caused by peripheral nerve injury, suggesting the involvement of P2X4 receptors ${ }^{[35]}$. Thus, blocking P2X4Rs in microglia may be a new therapeutic strategy for pain induced by nerve injury.

Studies in various CNS injury models suggest that activation of P2X7 receptors leads to neuronal death. Survival of retinal ganglion cultures declined with increasing exposure to $\mathrm{ATP}^{[36]}$. It is believed that this process is at least partially 
mediated by intracellular $\mathrm{Ca}^{2+}$ accumulation ${ }^{[37]}$. In vivo studies revealed that exposure to high levels of ATP led to irreversible increases in cytosolic calcium and cell death, and this effect is believed to be mediated through P2X7 receptors. P2X7 receptor antagonists could inhibit this excitotoxicity-based neuronal degeneration, reducing both the extent and functional sequelae of acute spinal cord injury ${ }^{[38]}$. Although numerous studies suggest that blockade of $\mathrm{P} 2 \mathrm{X} 7$ receptors may reduce excessive neuronal inflammation, work to date using $\mathrm{P} 2 \mathrm{X}^{-/-}$animals in an ischemic model did not confirm this effect. Clearly, further experiments are required to develop a consensus on the role of P2 receptors in CNS inflammation and to elucidate the crosslink between purinergic and adenosinergic signaling. For example, depression of synaptic transmission in the hippocampus during hypoxia was alleviated by A1 receptor agonists $^{[39]}$.

\section{Conclusion}

The immune system represents an important and unique defense mechanism in our body. Dysfunction of the immune response may lead to numerous diseases. In this sophisticated defense network, nucleotides play a recognized role in physiological and pathophysiological processes, such as chemotaxis, proliferation, cytokine secretion, cell death and intercellular communication. The pharmacological modulation of nucleotide-mediated signaling in cells represents a desirable new therapeutic approach for the treatment of inflammatory diseases and cancer. In recent years, several P2X receptor agonists were used to treat inflammatory diseases. The use of nonselective P2X receptor antagonists was proposed for the treatment of rheumatoid arthritis and pain induced by nerve injury. ATP was successfully applied to limit $\mathrm{T}$ cell-mediated inflammation in mouse models of type 1 diabetes and inflammatory bowel disease. Thus, purinergic receptor blockade might represent a promising therapeutic strategy for the treatment of a number of diseases. Although P2 holds great potential in the treatment of many immune diseases, it is essential for future investigations to understand how ATP affects the immune system mechanistically and to identify $\mathrm{ADP}^{\prime}$ s precise role in coordinating adenosine signaling. It is conceivable that new techniques such as fluorescence resonance energy transfer $(\text { FRET })^{[40]}$ and the real-time assessment of ATP release ${ }^{[41]}$ will help to elucidate the underlying mechanism of purinergic signaling and allow us to develop more specific and effective immuno-therapies.

\section{Acknowledgements}

Our study was supported by a grant (№ 30570757) from the National Natural Science Foundation of China. We thank Professor Fu-li YU, University of Illinois College of Medicine at Rockford, for reading this manuscript.

\section{References}

1 Ralevic V, Burnstock G. Receptors for purines and pyrimidines. Pharmacol Rev 1998; 50: 413-92.

2 Di Virgilio F, Ceruti S, Bramanti P, Abbracchio MP. Purinergic signaling in inflammation of the central nervous system. Trends Neurosci 2009; 32: 79-87.

3 Corriden R, Chen Y, Inoue Y, Beldi G, Robson SC, Insel PA, et al. Ectonucleoside Trisphosphate Diphosphohydrolase 1 (E-NTPDase 1/ CD39) regulates neutrophil chemotaxis by hydrolyzing released ATP to adenosine. J Biol Chem 2008; 283: 28480-6.

4 Virgilio FD, Borea PA, Illes P. P2 receptors meet the immune system. Trends Pharmacol Sci 2001; 22: 5-7.

5 Chen Y, Corriden R, Inoue Y, Yip L, Hashiguchi N, Zinkernagel A, et al. ATP release guides neutrophil chemotaxis via $\mathrm{P} 2 \mathrm{Y} 2$ and $\mathrm{A} 3$ receptors. Science 2006; 314: 1792-5.

6 Elliott MR, Chekeni FB, Trampont PC, Lazarowski ER, Kadl A, Walk SF, et al. Nucleotides released by apoptotic cells act as a find-me signal to promote phagocytic clearance. Nature 2009; 461: 282-7.

7 Bulanova E, Bulfone-Paus S. P2 receptor-mediated signaling in mast cell biology. Purinergic Signal 2010; 6: 3-17.

8 Davalos D, Grutzendler J, Yang G, Kim JV, Zuo Y, Jung S, et al. ATP mediates rapid microglial response to local brain injury in vivo. Nat Neurosci 2005; 8: 752-8.

9 Haynes SE, Hollopeter G, Yang G, Kurpius D, Dailey ME, Gan WB, et al. The $\mathrm{P} 2 \mathrm{Y} 12$ receptor regulates microglial activation by extracellular nucleotides. Nat Neurosci 2006; 9: 1512-9.

10 Koizumi S, Shigemoto-Mogami Y, Nasu-Tada K, Shinozaki Y, Ohsawa K, Tsuda M, et al. UDP acting P2Y6 receptors is a mediator of microglial phagocytosis. Nature 2007; 446: 1091-5.

11 Kettenmann H. The brain's garbage men. Nature 2007; 446: 987-8.

12 Elliott MR, Chekeni FB, Trampont PC, Lazarowski ER, Kadl A, Walk SF, et al. Nucleotides released by apoptotic cells act as a find-me signal to promote phagocytic clearance. Nature 2009; 461: 282-7.

13 Atarashi K, Nishimura J, Shima T, Umesaki Y, Yamamoto M, Onoue $\mathrm{M}$, et al. ATP drives lamina propria TH17 cell differentiation. Nature 2008; 455: 808-12.

14 Di Virgilio F, Robson SC. Identification of novel immunosuppressive pathways paves the way for drug discovery. Curr Opin Pharmacol 2009; 9: 445-6.

15 Boeynaems JM, Communi D. Modulation of inflammation by extracellular nucleotides. J Invest Dermatol 2006; 126: 943-4.

16 Yip L, Woehrle T, Corriden R, Hirsh M, Chen Y, Inoue Y, et al. Autocrine regulation of T-cell activation by ATP release and $\mathrm{P} 2 \mathrm{X} 7$ receptor. FASEB J 2009; 23: 1685-93.

17 Idzko M, Hammad H, van Nimwegen M, Kool M, Willart MA, Muskens $\mathrm{F}$, et al. Extracellular ATP triggers and maintains asthmatic airway inflammation by activating dendritic cells. Nat Med 2007; 13: 913-9.

18 Dinarello CA. Immunological and inflammatory functions of the interleukin-1 family. Annu Rev Immunol 2009; 27: 519-50.

19 Al-Shukaili A, Al-Kaabi J, Hassan B. A comparative study of interleukin-1 $\beta$ production and P2X7 expression after ATP stimulation by peripheral blood mononuclear cells isolated from rheumatoid arthritis patients and normal healthy controls. Inflammation 2008; 31: 84-90.

20 Hewinson J, Moore SF, Glover C, Watts AG, Mackenzie AB. A key role for redox signaling in rapid $\mathrm{P} 2 \mathrm{X} 7$ receptor-induced IL-1 $\beta$ processing in human monocytes. J Immunol 2008; 180: 8410-20.

21 Di Vigilio F, Boeynaems JM, Robson SC. Extracellular nucleotides as negative modulators of immunity. Curr Opin Pharmacol 2009; 9: 507-13.

22 Di Virgilio F. Liaisons dangereuses: P2X7 and the inflammasome. Trends Pharmacol Sci 2007; 28: 465-72.

23 Pelegrin P, Surprenant A. Pannexin-1 mediates large pore formation and interleukin-1beta release by the ATP-gated P2X7 receptor. EMBO J 2006; 25: 5071-82. 
24 Deaglio S, Dwyer KM, Gao W, Friedman D, Usheva A, Erat A, et al. Adenosine generation catalyzed by CD39 and CD73 expressed on regulatory T cells mediates immune suppression. J Exp Med 2007; 204: 1257-65.

25 Burnstock G. Pathophysiology and therapeutic potential of purinergic signaling. Pharmacol Rev 2006; 58: 58-86.

26 Deli T, Csernoch L. Extracellular ATP and cancer - An overview with special reference to P2 purinergic receptors. Pathol Oncol Res 2008; 14: 219-31.

27 Maaser K, Höpfner M, Kap H, Sutter AP, Barthel B, von Lampe B, et al. Extracelluar nucleotides inhibit growth of human oesophageal cancer cells via P2Y2 receptors. Br J Cancer 2002; 86: 636-44.

28 Solle M, Labasi J, Perregaux DG, et al. Altered cytokine production in mice lacking P2X7 receptors. J Biol Chem 2001; 276: 125-32.

29 White N, Burnstock G. P2 receptors and cancer. Trends Pharmacol Sci 2006; 27: 211-7.

30 Fields RD, Burnstock G. Purinergic signaling in neuron-glia interactions. Nat Rev Neurosci 2006; 7: 423-36.

31 Seman M, Adriouch S, Scheuplein F, Krebs C, Freese D, Glowacki G, et al. NAD-induced T cell death: ADP-ribosylation of cell surface proteins by ART2 activates the cytolytic P2X7 purinoceptor. Immunity 2003; 19: 571-82.

32 Kucher BM, Neary JT. Bi-functional effects of ATP/P2 receptor activation on tumor necrosis factor- $\alpha$ release in lipopolysaccharidestimulated astrocytes. J Neurochem 2005; 92: 525-35.

33 Wang CM, Chang YY, Kuo JS, Sun SH. Activation of P2X7 receptors induced $\left[{ }^{3} \mathrm{H}\right] \mathrm{GABA}$ release from the RBA-2 type-2 astrocyte cell line through a $\mathrm{Cl}^{-} / \mathrm{HCO}_{3}$-dependent mechanism. Glia 2002; 37: 8-18.

34 Parvathenani LK, Tertyshnikova S, Greco CR, Roberts SB, Robertson B, Posmantur R. P2X7 mediates superoxide production in primary microglia and is up-regulated in a transgenic mouse model of Alzheimer's disease. J Biol Chem 2003; 278: 13309-17.

35 Tsuda M, Shigemoto-Mogami Y, Koizumi S, Mizokoshi A, Kohsaka S, Salter MW, et al. P2X4 receptors induced in spinal microglia gate tactile allodynia after nerve injury. Nature 2003; 424: 778-83.

36 Zhang X, Zhang M, Laties AM, Mitchell $\mathrm{CH}$. Stimulation of P2X7 receptors elevates $\mathrm{Ca}^{2+}$ and kills retinal ganglion cells. Invest Ophthalmol Vis Sci 2005; 46: 2183-91.

37 Anderson CM, Nedergaard M. Emerging challenges of assigning P2X7 receptor function and immunoreactivity in neurons. Trends Pharmacol Neurosci 2006; 29: 257-62.

38 Wang X, Arcuino G, Takano T, Lin J, Peng WG, Wan P, et al. P2X7 receptor inhibition improves recovery after spinal cord injury. Nat Med 2004; 10: 821-7.

39 Fowler JC, Gervitz LM, Hamilton ME, Walker JA. Systemic hypoxia and the depression of synaptic transmission in rat hippocampus after carotid artery occlusion. J Physiol (Lond) 2003; 550: 961-72.

40 Richler E, Chaumont S, Shigetomi E, Sagasti A, Khakh BS. Tracking transmitter-gated $\mathrm{P} 2 \mathrm{X}$ cation channel activation in vitro and in vivo. Nat Methods 2008; 5: 87-93.

41 Corriden R, Insel PA, Junger WG. A novel method using fluorescence microscopy for real-time assessment of ATP release from individual cells. Am J Physiol Cell Physiol 2007; 293: C1420-5. 